\title{
Using field strength scaling to save energy in mobile HF-band RFID-systems
}

\author{
Manuel Menghin ${ }^{1 *}$, Norbert Druml ${ }^{1}$, Christian Steger ${ }^{1}$, Reinhold Weiss ${ }^{1}$, Holger Bock ${ }^{2}$ and Josef Haid ${ }^{2}$
}

\begin{abstract}
Radio frequency identification (RFID) is a technology enabling a contactless exchange of data. This technology features the possibility to wirelessly transfer power to the transponder (opponent). HF-RFID is used in mobile devices like smart phones and shows potential for applications like payment, identification, etc. Unfortunately, the needed functionality increases the battery drain of the device. As a countermeasure, power-management techniques are implemented. However, these techniques commonly do not consider the whole system, which also consists of the communication to the transponder, to prevent wasting energy. One cross-system technique of reducing the wasted energy is magnetic field strength scaling, which regulates the power transfer to the transponder. This article shows three investigations made, regarding field strength scaling to prevent this wastage of energy. The results of one investigation, how to use field strength scaling at card detection phase in form of the PTF-Determinator method, is described in detail. This method determines the Power Transfer Function (PTF) during run-time and scales the provided power accordingly to save energy. As a case study the PTF-Determinator is integrated in an application to read digital business cards. The resulting power consumption and timing has been evaluated by simulation and measurement of a development platform for mobile phones. Furthermore, the impact of field strength scaling to the energy consumption of a state of the art NFC-enhanced smart phone has been analyzed. The results of the case study shows that up to $26 \%$ less transmission energy (energy drain of NFC) is needed, if field strength scaling is applied (proofen by measurement). According to this result a smart phone's battery drain (energy drain of the whole system) can be decreased by up to $13 \%$ by using field strength scaling for this case study.
\end{abstract}

Keywords: RFID, NFC, Power consumption, Magnetic field strength scaling, Power transfer, Power-management

\section{Introduction}

HF-band radio frequency identification (RFID) is a wireless form of communication. One feature of this wireless communication form is the possibility to transfer power from the reader (sender) to the transponder (receiver). There are many standards using this communication form. One of them is near field communication (NFC). An exemplary application of NFC is using it in mobile devices like smart phones, which opens a wide set of applications like payment, identification, and ticketing. Unfortunately, NFC increases the battery drain, because of the additional power-consumption needed by the reader during communication. Minimizing this consumption is the goal of the power-management algorithms implemented in software

\footnotetext{
*Correspondence: manuel.menghin@tugraz.at

${ }^{1}$ Graz University of Technology, Graz, Austria

Full list of author information is available at the end of the article
}

and hardware. These algorithms commonly focus on one component and do not consider the whole system.

The considered target system consists of multiple components as shown in Figure 1. The mobile RFID-Reader includes a battery, which can only provide a limited amount of energy. The reader also has to power the Reader-IC needed for the RFID communication. The transponder has no own power source and is powered over RF. This wireless power transfer includes losses (more power has to be provided by the reader to satisfy the power requirements of the transponder). This discussed RFID communication (HF-Band) is based on inductive coupling where an alternating magnetic field is used to transfer the data and the power to the transponder.

The reader is able to control this transferred power by scaling the strength of the magnetic field. In most cases, the provided power by the reader is set to a maximum value to ensure the transponder's proper supply at an

\section{照 Springer}

(c) 2013 Menghin et al: licensee Springer. This is an Open Access article distributed under the terms of the Creative Commons Attribution License (http://creativecommons.org/licenses/by/2.0), which permits unrestricted use, distribution, and reproduction in any medium, provided the original work is properly cited. 


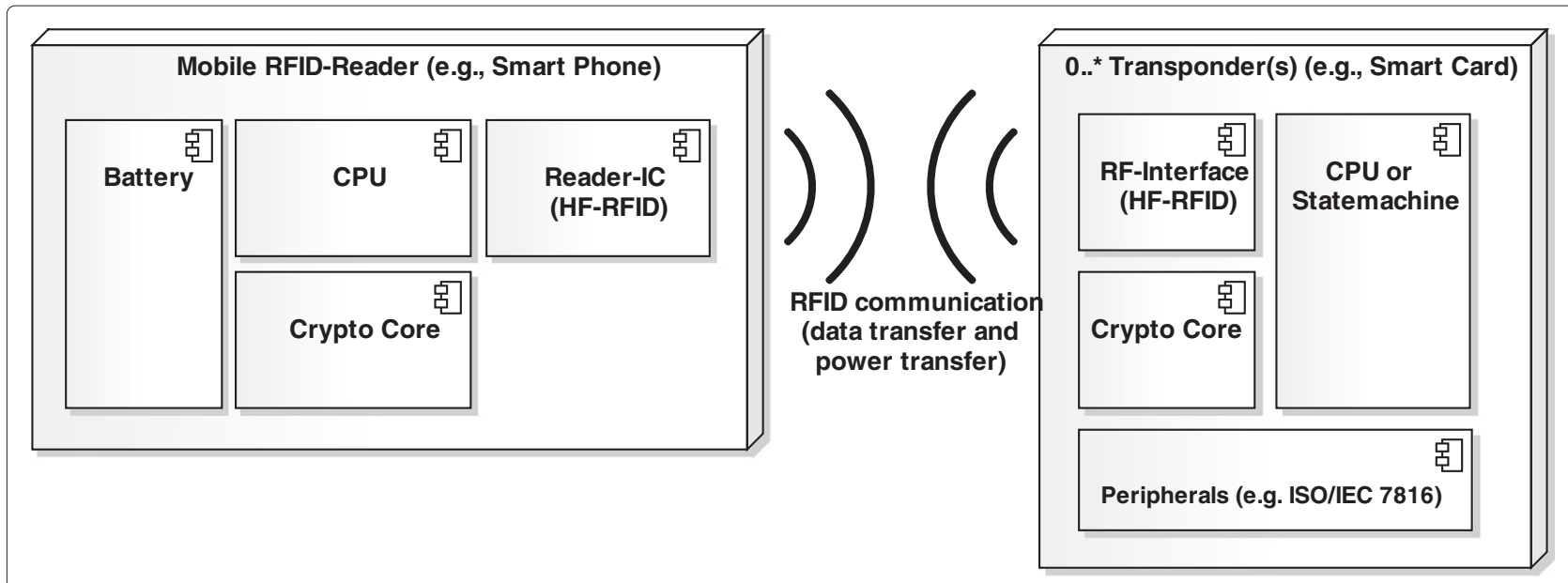

Figure 1 Target system. This component model describes the target system considered in this article. Basically, it consists of the battery-powered HF-RFID reader and zero to multiple transponder(s). The case study of this article considers the case of one reader connected to one transponder.

expected transmission distance (about $5-10 \mathrm{~cm}$ ) regardless of the transponder-type. This provided power output is in most cases (e.g., closer distance) too high and leads to an oversupply of the transponder. To give an example, the power consumption of a state-of-the-art smart phone with NFC has been measured, which is shown in Section 5.4 in detail. The result shows that the smart phone needs $50 \%$ more power when NFC is activated but no transponder is in range compared to the consumption without NFC. However, if a transponder is read over NFC, then the device consumes up to $107 \%$ more power. This shows that reducing the power consumption of NFC plays a relevant role in decreasing the battery drain of the RFIDReader (e.g., smart phone). One way to achieve that is the dynamic configuration of the magnetic field strength by the reader during run-time. To perform this dynamic configuration, the reader has to know, which field strength is currently needed to supply the transponder properly. The investigations made to realize this dynamic configuration can be divided into three parts:

- The first investigation regards dynamic field strength scaling during card detection phase. The challenge lies in gathering the needed parameters (e.g., distance between reader and transponder) to evaluate the power transfer function (PTF) after a transponder has been detected. Based on the PTF knowledge, the reader is able to properly scale the magnetic field strength. Additional methods to prevent a wastage of energy can be implemented based on the knowledge of the PTF, which will be discussed later on [1].

- During the phase of RFID communication, the distance between reader and transponder can change (user typically moves the transponder in the direction to the reader), which results in a changing PTF. Furthermore, different operations, like reading data from the transponder or performing an encryption, lead to a change of the transponder's power requirements. The adaption of the magnetic field strength during communication is important, to preserve a proper supply for the transponder at all time [2].

- The RFID technology is able to read multiple transponders in range. Multiple transponders in the magnetic field influence the PTF, which also depends on the states of these transponders (e.g., selected and active or deselected). These dependencies occur because different transponder states provoke different power consumption requirements. With this knowledge, the PTF can be redetermined and the consumed energy can be reduced by a proper field strength scaling algorithm.

The contribution of this article consists of three parts:

- Three investigations of magnetic field strength scaling in HF-Band RFID-Systems to create a power-aware system are presented.

- The investigation how to use field strength scaling during card detection phase in form of the novel PTF-Determinator method is described in detail in [1] (see Figure 2).

- A case study using the PTF-Determinator in an application of reading digital business cards (including a feature to restrict the maximum transmission distance) is shown [1].

The remainder of this article is split into five main parts. The first part can be found in Section 2, which shows the related work and highlights the contribution. The second part shows the three investigations to realize magnetic field strength scaling. As third part, which can be found in Section 4, the PTF-Determinator and its integration into 


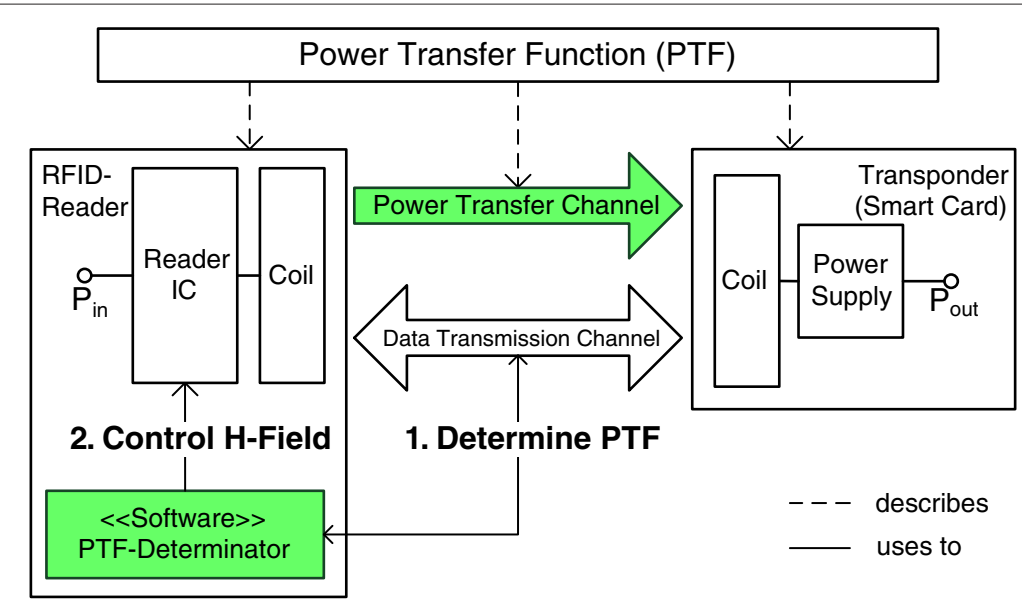

Figure 2 The proposed first step towards field strength scaling using the PTF-Determinator and the integration into the NFC system.

the RFID-System is shown in detail. The case study is presented in the fourth part in Section 5. The fifth part in Section 6 finally concludes this article.

\section{Related work}

This section is split into four parts. The first part deals with the state-of-the-art possibilities to acquire the physical relation factor (simplified the distance between reader and transponder). The second part shows investigations regarding the influence of the transponder's operation to it's power requirements. In addition, the related work regarding multiple transponders is shown in part three. In the fourth part, known system-based power-management concepts including reader and transponder are shown.

\subsection{Acquisition of the physical relation factor}

One consideration regards acquiring the physical relation, like the distance between the reader and the transponder coil, and other dynamic parameters, during run-time. An approach is to find a known parameter that describes this physical relation. Cheng et al. [3] show that there is a relation between the provided power of the reader and the distance to the transponder. The analysis has been concluded by altering the signal strength of the reader and checking if the transponder has enough power to be active. Another approach is distance bounding, which uses the delay between the request and the response as known parameter to calculate the physical distance between reader and transponder to detect relaying attacks [4]. To use this information to determine the PTF, the parameter has to be measured during run-time. Furthermore, transmission characteristics (e.g., coil dimensions) have to be included into the determination. These characteristics depend on the system's setup (e.g., different types of transponders). Xu et al. [5] use power stepping to detect different positioned transponders (distance to the reader). This consideration does not include the physical principles of the power transfer but leads to a evaluation of a parameter, which is similar to a distance value, during run-time. Another method measures the voltage on transponder side and to use it for the PTF determination [6].

\subsection{Power requirements of the passive transponder}

Another fact to consider is that the transponder is only passively powered by the reader [7]. This means that the transponder cannot respond, if the provided power drops under a certain threshold. Furthermore, the power consumption of the transponder itself depends on the currently executed operation, which influences the level of needed power [8]. Power-consuming operations are especially encryptions/decryptions [9]. Mercier et al. [10] show the relation between the provided power and the consumption of the circuit. To consider this in the determination of the PTF, the transponder has to be in a state that is aware of its power consumption. The last point of consideration is the transmission of the data (response), which is realized through load modulation on transponder side. The influence on the modulation is similar to the power consumption of the transponder [11]. One possibility to acquire the power consumption of the transponder during run-time is using the principle of using power estimation units directly on the transponder. This information can be transferred to the reader afterwards [12].

\subsection{Influence of multiple transponders on the PTF}

Multiple transponders in RF field influence the power consumption of the RFID-System, and a PTF used for one transponder is not enough to describe this environment. Collisions for example influence the power consumption of the total system. The reader sends a request and gets the response from the transponders. These messages can 
collide and have to be detected. There is a protocol included in the detection algorithm to deal with this challenge. Most of the publications made in this topic are dealing with the question, how to optimize or avoid these collisions. Kamineni et al. [13] are using the power level information on transponder side to avoid collisions by defined delays according this power level. Other published approaches deal with the avoidance of collisions to increase the performance of the system $[14,15]$.

\subsection{System-based power-management for RFID}

Liu and Tong [16] describe energy provisioning services. They show that knowing the system can lead to optimization possibilities. Their concept focuses on a multi-tag multi-reader application but it can be adapted to the challenge of field strength scaling. This knowledge can be used to optimize the system in terms of power consumption and stability. This should especially be considered in combination with mobile readers [17]. The challenge is to manage the distributed information and the calculation among the system for power-optimization. The Cinder operating system is an example how such optimizations can be done. This approach is designed for smart phones but the model can be extended to include externally powered devices as well [18].

\section{Investigations made for field strength scaling}

This section describes the three investigations made to use magnetic field strength scaling in RFID-Systems as mentioned in Section 1. These investigations are separated according to the overview of the RFID communication flow and the dependencies to the PTF as shown in Figure 3. The reader should know the PTF at each point in time to react on changes. The first investigation regards the determination of the PTF during card detection phase. The second investigation deals with the dependencies to the currently executed operation invoked by the reader and sent to the transponder (e.g., read block), and the changing physical relation factor (distance) of the transponder. The third investigation regards the influence of the multiple transponders in range to the PTF.

\subsection{Field strength scaling during card detection phase}

If a transponder gets in range, the reader is able to establish the communication by sending the request (e.g., REQA) and an anti-collision command (needed for multitransponder communications). The transponder answers to this anti-collision command with its unique identification number (UID). This procedure is followed by a selection command, which elevates the transponder to the ready state. During this card detection phase, data are already exchanged between the reader and the transponder. If this phase is modified to determine the PTF, the reader would be able to scale the magnetic field strength accordingly before the communication process begins (e.g., reading a digital business card). The challenge of this approach is getting the information needed for the determination during this phase (a detailed description is given in Section 4) [1]. One issue, which is not covered by this approach, is the changing physical relation factor during communication. An initial magnetic field scale can lead to an oversupply or to an undersupply of the transponder during the communication process.

\subsection{Dynamic field strength scaling during communication}

This approach deals with the issue of dynamically scaling the field strength during communication. A scenario can scaling the field while reading the data of a digital business card. Thus, this method avoids an oversupply or undersupply by inappropriate scaling. The challenge of this approach can be split into two parts. The first part is detecting changes of the physical relation factor (distance) during communication. These changes occur by pulling the transponder from or pushing it towards the reader, which can be a scenario (e.g., access card is held by the user against the reader). Redeterming the PTF using the same method as in the card detection would result in a considerable communication overhead. This redetermination has to be made fast enough to react on the change of the physical relation factor. Thus, another way has to be found to detect the changes like finding an easy to acquire equivalent parameter to the physical relation factor on reader side. The second part concerns the fact that different transponder commands demand different transponder power requirements, e.g., reading a memoryblock versus performing cryptographic operations. This also has to lead to a redetermination of the PTF and to a proper scaling [2].

\subsection{Field strength scaling for multiple transponders}

The two described ways of accomplishment do not consider multiple transponders in range. The usage of multiple transponders with one reader is not restricted to logistic applications, like reading tagged packets. An example of other applications is a briefcase, with multiple access cards. These transponders influence the PTF in two ways. First, each transponder has a different physical relation factor (distance to the reader). The transponders cannot be on the same position. The PTF depends on the currently selected transponder. Second, the instantaneous power consumption of a transponder influences the PTF of all other transponders. Therefore, the reader has to be aware of the presence of all transponders and their states to properly scale the magnetic field to avoid an oversupply or undersupply. Furthermore, a policy has to be defined how to interact with the transponders. The transponders, which are not selected, can either get a proper supply to remain in their states, or the policy allows the undersupply 


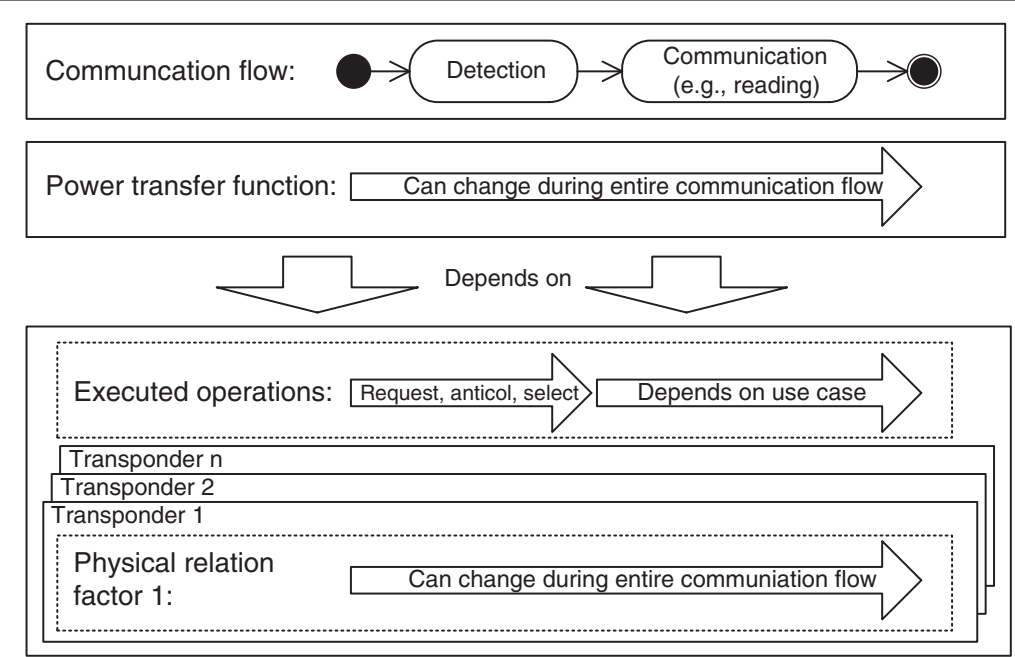

Figure 3 Overview of the dependencies of the PTF during communication. This figure gives an overview of the dependencies during communication regarding the PTF. The upper part visualizes the communication flow. The lower two parts are the dependencies of the PTF during the communication.

of those deselected transponders to save energy with the disadvantage of a loss of connection to them.

\section{Method - Field strength scaling during card detection in detail}

In this Section, the investigation of field strength scaling during card detection phase and the used method called PTF-Determinator is shown. The challenge to determine the PTF in the card detection phase is to collect the needed information during this phase and to pass this information to the reader side. With the determined PTF, the field strength can dynamically be scaled. To realize the above described steps, the RFID-System has to be defined and examined. For examination, the target system is reduced to one reader and one transponder. The RFID communication channel between them is split into two main parts. The first part is the power transmission path. It describes how the provided power, which can be altered by the reader, is transferred to the transponder. The second part is the communication channel used to exchange data between reader and transponder. The solution how to integrate the PTF-Determinator method into an existing RFID-System is shown in Figure 2.

At first, the needed electrical characteristics for the realization of the method are shown. The realization itself is split into four considerations. The first one deals with gathering the needed parameters from the reader and transponder. The second relates to the evaluation of the physical relation factor, which cannot be acquired directly. The third consideration deals with the integration of the method into the RFID-System's existing communication flow. The fourth consideration describes a library providing an interface to access the determined
PTF. This library can be used for power-management methods.

\subsection{Electrical characteristics}

This section explains the used replacement circuit and equations for the method to calculate the PTF. The equations describe how the power is transferred from the Reader-IC to the supply of the transponder. The replacement circuit describes the connection between them, as shown in Figure 4. The calculation is split into four parts.

The first part describes the power control of the ReaderIC, which can be configured by a resistance $\left(R_{\text {rel }}\right)$ serial to a constant voltage-source $\left(U_{1}\right)$ as shown in Equation (1).

$$
i_{r}=\frac{U_{1}}{Z_{c}+R_{\text {rel }}}
$$

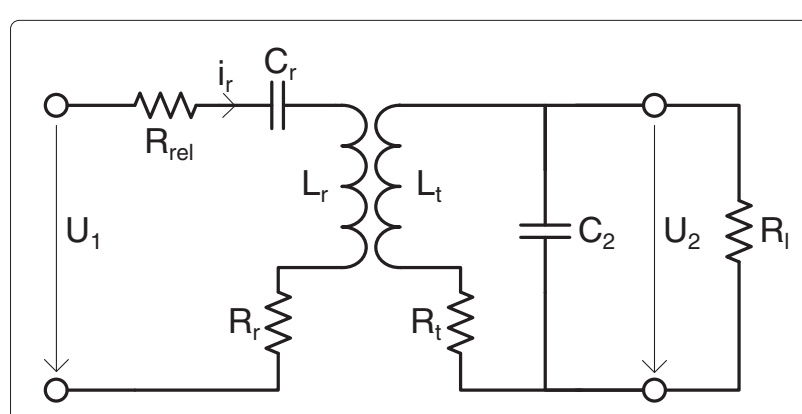

Figure 4 Replacement circuit of the power transfer over RFID. This replacement circuit describes the power transfer from the reader to the transponder without the voltage regulation on transponderside, adapted from [19]. In this case, the input current $i_{r}$ is scaled by the resistance $R_{\text {rel }} U_{2}$ represents the supply voltage of the transponder. 
Increasing the resistance causes a reduction of the overall power (decrease of $i_{r}$ ) consumption with the disadvantage of loosing transmission range [20]. The current $i_{r}$ also depends on the configurable resistance $R_{\text {rel }}$ and the input resistance $Z_{c}$ of the circuit beyond. $Z_{c}$ alters according to the inductive coupling between reader and transponder and is therefore not static.

The second part consists of the equation used to calculate the provided magnetic field $\mathrm{H}$ of the reader, which is provoked by the electrical current $i_{r}$. The considered orientation of the sender and receiver coil is shown in Figure 5.

Equation (2) can be used for rectangular-shaped sender coils and is based on the law of Biot-Savart. It is based on the physical principle of loose inductive coupling. The needed parameters are the dimensions $a_{r}$ and $b_{r}$ of the reader coil and the number of windings $N_{r}$. The distance to the coil can only be used if the coils are coaxial oriented [19].

$$
\begin{aligned}
H= & \frac{i_{r} \cdot N_{r} \cdot a_{r} \cdot b_{r}}{4 \cdot \pi \cdot \sqrt{\left(\frac{a_{r}}{2}\right)^{2}+\left(\frac{b_{r}}{2}\right)^{2}+d^{2}}} \\
& \cdot\left(\frac{1}{\left(\frac{a_{r}}{2}\right)^{2}+d^{2}}+\frac{1}{\left(\frac{b_{r}}{2}\right)^{2}+d^{2}}\right)
\end{aligned}
$$

The third part deals with the transformation of the magnetic field strength back to a voltage on transponder side (see Figure 4). A resonance circuit, consisting of a parallel capacitance and the coil's inductance, is used to amplify the received voltage. First of all the coupling coefficient $k$ is calculated with Equations (3) and (4). The coefficient represents an abstract relation between reader and transponder and requires the magnetic field strength as input. Equation (5) calculates the resulting voltage on transponder-side. This is only valid for rectangular receiver coils. The needed input parameters are the

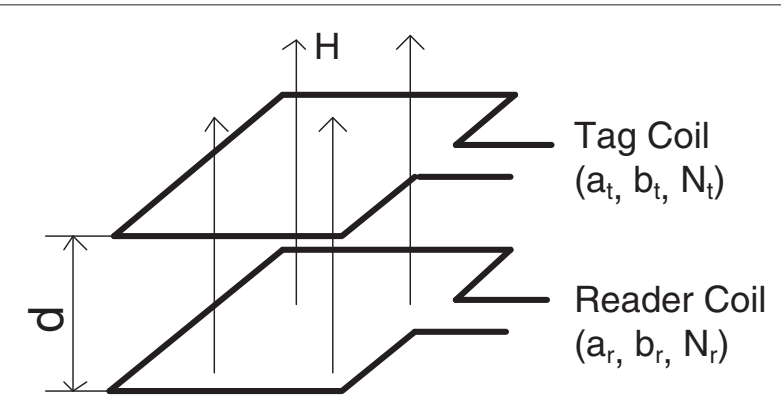

Figure 5 Considered coaxial orientation of coils. This figure visualizes the considered coaxial orientation of the reader to the transponder by using rectangular shaped sender and receiver coils. dimensions $a_{t}$ and $b_{t}$, the number of windings $N_{t}$, and the coil's inductance $L_{t}[19]$.

$$
\begin{aligned}
& M_{12}=\frac{\mu_{0} \cdot H \cdot N_{t} \cdot a_{t} \cdot b_{t}}{i_{r}} \\
& k=\frac{M_{12}}{\sqrt{L_{r} \cdot L_{t}}} \\
& u_{2}=\frac{w \cdot k \cdot \sqrt{L_{r} \cdot L_{t}} \cdot i_{r}}{\sqrt{\left(\frac{\left(w \cdot L_{t}\right)}{R_{l}}+w \cdot R_{t} \cdot C_{2}\right)^{2}+\left(1-w^{2} \cdot L_{t} \cdot C_{2}+\frac{R_{t}}{R_{l}}\right)^{2}}}
\end{aligned}
$$

The fourth part describes that the output voltage is limited by a Zener-diode to prevent the smart card's electronics from power surges. It is also necessary to provide a minimum voltage. If the supply drops below the minimum threshold voltage, the circuit is set to power down. Figure 6 shows an example of the relation between reader/transponder distance, and the supply voltage of the transponder (the power consumption of the transponder is considered static) for several reader power output levels.

\subsection{Gathering needed parameters}

As first consideration, the needed parameters have to be collected from the RFID-System. They are distributed between reader and transponder. Some of them are physical values, which have to be stored in digitalized form. Because of the system's variability during run-time (e.g., different transponders), the storage of all parameters in a single location is inappropriate. Table 1 depicts the number of parameters, their location, and their required space.

These parameters shall be provided on the described location shown in Figure 7. One approach is to store this data into a memory during the device's production phase

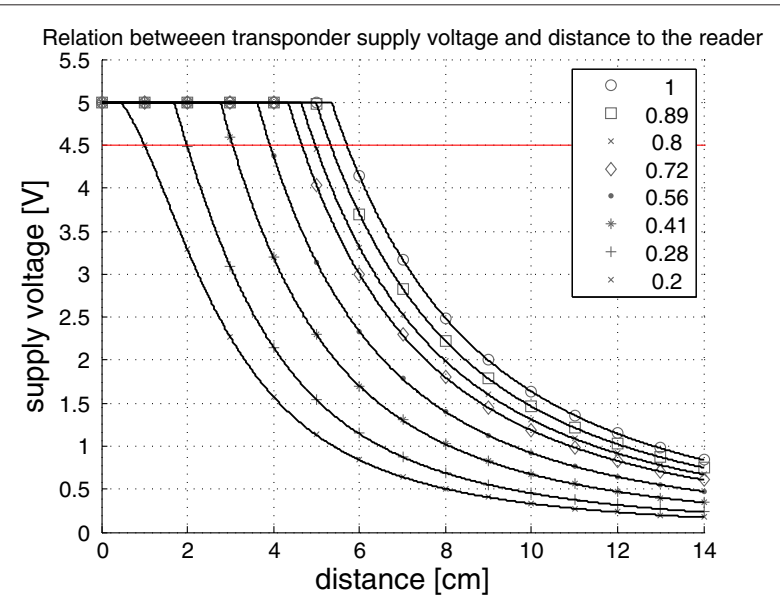

Figure 6 Relation to the supply voltage of the transponder. Plot of the relation between the supply voltage of the transponder to the distance between the reader and the transponder. The relation is shown using different input power levels as described in the legend. The two coils are oriented coaxial. 
Table 1 Number of parameters needed for the PTF-Determinator including their location and needed space in bytes

\begin{tabular}{lccc}
\hline Location & $\begin{array}{c}\text { Number of } \\
\text { parameters }\end{array}$ & $\begin{array}{c}\text { Accuracy } \\
\text { [b] }\end{array}$ & $\begin{array}{c}\text { Space } \\
\text { [B] }\end{array}$ \\
\hline Mobile RFID-Reader & 5 & 32 & 20 \\
Transponder & 8 & 8 & 5 \\
& & 32 & 32 \\
\end{tabular}

The space is shown with different accuracies of the parameters.

(reader and transponder). The needed storage space is 32 byte on transponder-side if an accuracy of 32 bit is used. In practice, the needed storage can be decreased by adapting the resolution of the values according to the needed PTF accuracy requirements. An example is shown in Table 2 where the needed space is reduced to 8 bytes. These values have to be transferred to a central computation unit. This can either be the reader or the transponder. In this study, the reader has been chosen because of its advanced computational resources and a direct control of the needed input parameters for the PTF (parameter of provided power). This also means that the parametervalues from the transponder have to be transferred to the reader which can costly be in terms of time and power. For comparison, sending a ping request to the transponder requires 7-bit and results in a 2-byte response [19]. The request could be the same size but the response would be 16 times greater. If the bit length of the send values is reduced to 8 bits, which should be enough in practice, the bytes to sent can be four times greater.

\subsection{Evaluation of the physical relation factor}

The second consideration regards the physical relation factor, which is independent from the type of reader and transponder used. Furthermore, its value is unknown and has to be evaluated during run-time. It cannot directly be measured because of the lack of sensing mechanisms on both sides.
Table 2 Comparison of the saved energy for the RFID-transmission in percent between the method with and without an improved version of the PTF-Determinator from [1] in the simulation

\begin{tabular}{lccc}
\hline $\begin{array}{l}\text { Simulated } \\
\text { distance } \\
(\mathbf{c m})\end{array}$ & $\begin{array}{c}\text { Energy } \\
\text { with PTF } \\
\text { (Norm.) }\end{array}$ & $\begin{array}{c}\text { Energy } \\
\text { no PTF } \\
\text { (Norm.) }\end{array}$ & $\begin{array}{c}\text { Energy } \\
\text { saved } \\
\text { (\%) }\end{array}$ \\
\hline $0-1$ & 0.202 & 1.000 & 79.78 \\
$1-2$ & 0.263 & 1.000 & 73.75 \\
$2-3$ & 0.391 & 1.000 & 60.92 \\
$3-4$ & 0.674 & 1.000 & 32.59 \\
$4-5 x>x_{\max }$ & 0,072 & 1.000 & 92.78 \\
$5-6 x>x_{-\max }$ & 0,067 & 1.000 & 93.33 \\
\hline
\end{tabular}

To solve this problem, the PTF described in Section 4.1 is used. The equations of the PTF described in Section 4.1 have to be transformed to determine the physical relation using the provided power by the reader and the corresponding supply voltage of the transponder as input parameters. The supply voltage is also unknown because of the lack of an integrated sensor at transponder side. To approximate this value the current power state of the transponder can be used. This means if the transponder is not responding, the supply voltage is too low for operation $\left(<U_{t}\right)$. Otherwise, the transponder responds that the operation voltage is above the needed one $\left(>U_{t}\right)$ as shown in Figure $6\left(U_{t}\right.$ is marked as red line). If the reader's provided power is altered until the transition from power down to idle state is reached, the value of the supply voltage from the transponder is slightly above $U_{t}$ (see Figure 8).

The execution time needed for the approximation depends on the selected resolution of the power steps on reader-side. To use this method in practice, a balance between the power step resolution and the execution time needed for the algorithm has to be found. In case of ten steps this would also mean that four iterations have to be made with a successive approximation approach $\left(2^{n}\right)$. This

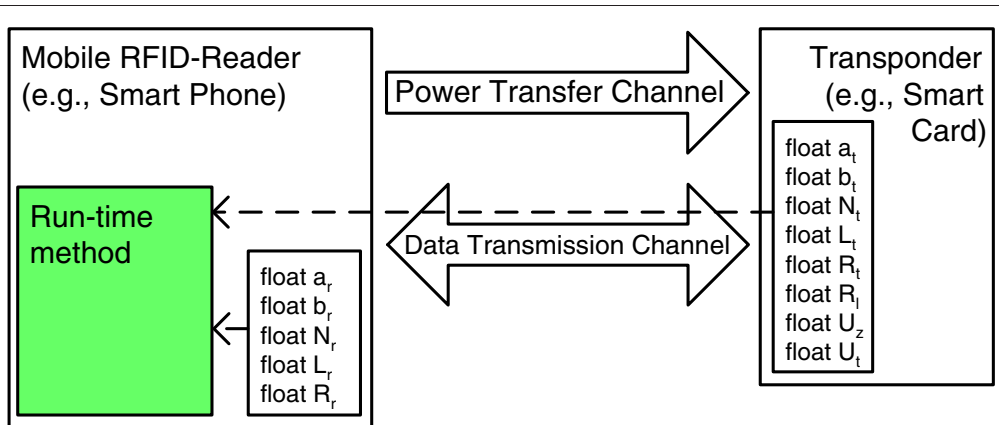

Figure 7 Distribution of the needed parameters. Distribution of the needed parameters to determine the PTF in the RFID-System and the possible paths to transfer them to a central location. 


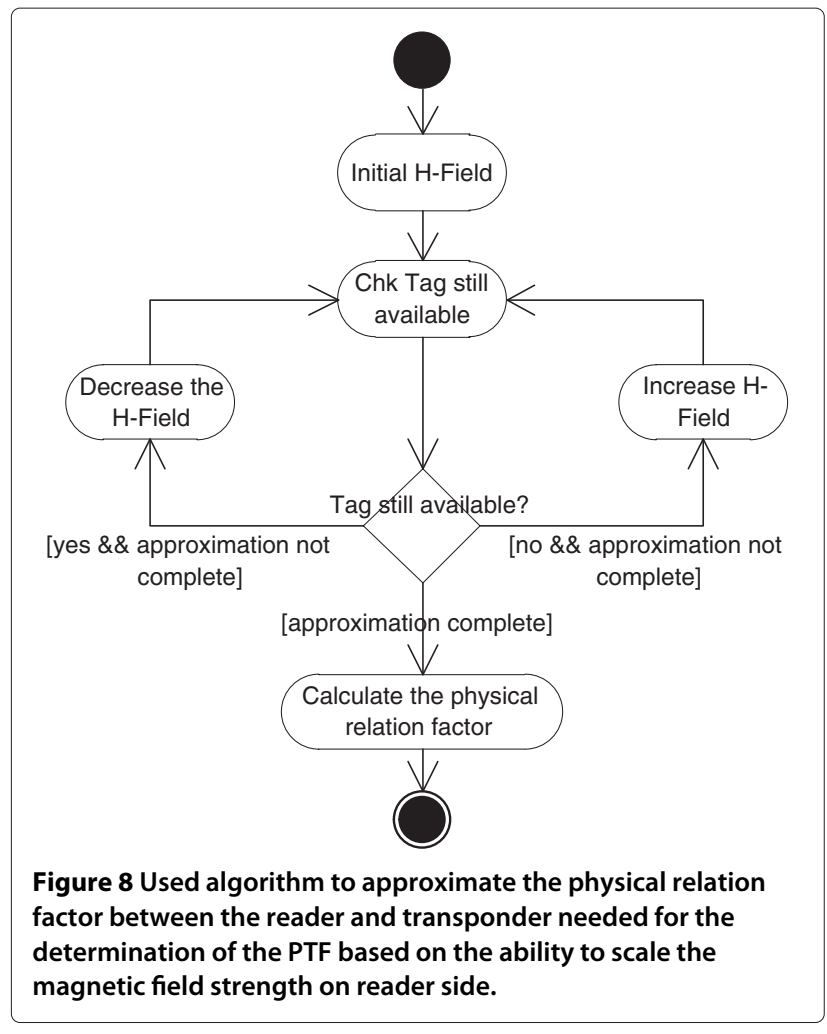

leads to a longer time needed for the whole execution and an increase of needed energy compared to a simple card detection phase. To keep this overhead as small as possible the operation to proof if the transponder is responding (state "Chk Tag still available" as shown in Figure 8) should only invoke a small response and computation-effort for the transponder, which would otherwise lower the benefit of field strength scaling to save energy. In this approach, the request command (REQA) to the transponder can be used.

\subsection{PTF-Determinator flow integration}

The third consideration deals with the inclusion of the PTF-Determinator into the existing communication flow of reader and transponder. The transponder supports different states, which influence the accepted commands from the transponder (see Figure 9).

When the transponder receives enough power it switches to idle state. In this state, a request from the reader is expected. After the request is issued it is possible to select the card by sending an anti-collision command followed by a select command with the appropriate UID. After this procedure, the transponder enables extended commands like reading values from the memory. This read command is needed by the PTF-Determinator. If the power supply drops below a certain threshold (e.g., by exceeding the maximum transmission distance between reader and transponder) the state is set back to

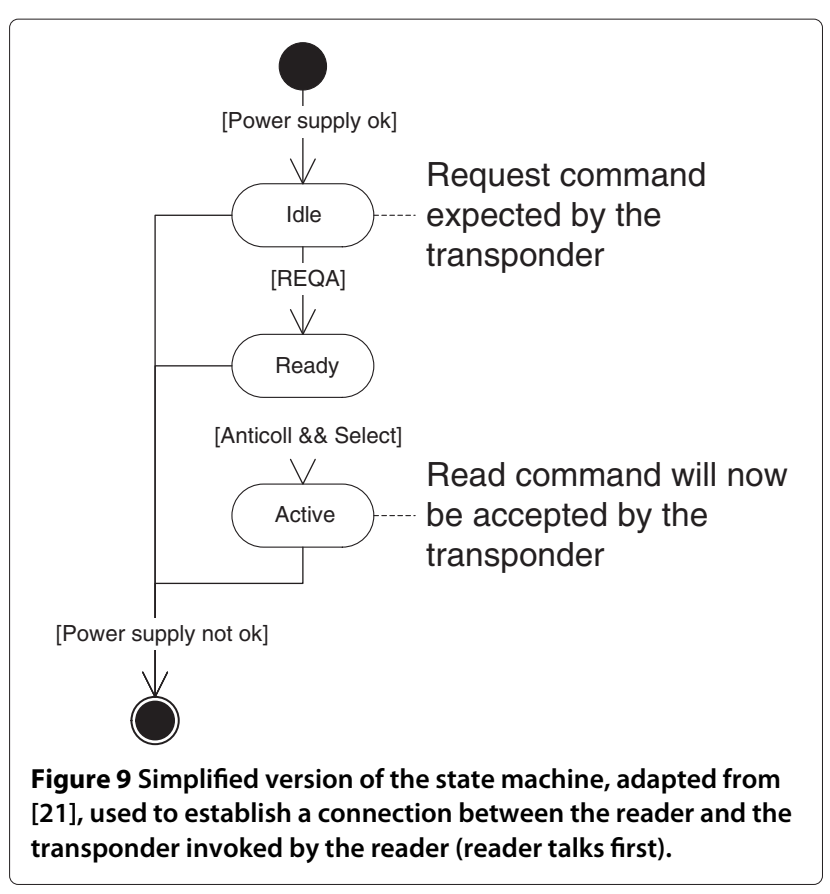

power down. To return to the active state the navigation through the state-machine of the transponder by sending a request (e.g., REQA) and select command has to be redone.

The integration of the PTF-Determinator method into the existing flow is split into three parts. The first part is executing the approximation algorithm as shown in Figure 8, but without calculating the physical relation factor. This approximation does not need any parameter information of the transponder, only a specified command to call. This can be REQA, which leads to a response which can be used to find out if the transponder is available or not. The second part is gathering the needed parameters from the transponder as shown in Figure 7, which needs to select the card to enable the command for reading. The third part is responsible for calculating the physical relation factor based on the gathered information from the other two parts. All needed information is now available to determine the PTF.

As last step of integration, it has to be defined, in which communication phase the PTF-Determinator is executed. As a first approach the method is included into the card detection phase. If a new transponder has been detected, the algorithm begins to determine the PTF, as described in the last paragraph, and is locked for operation until the method is finished. After that, the transponder is set to ready state and the wanted operations can be executed. Thanks to this approach, the knowledge of the PTF can be used after the card detection phase. Unfortunately, the time needed for this card detection phase increases. 
Furthermore, changes to the PTF after this phase cannot be detected.

\subsection{PTF library integration}

The last consideration is to provide the determined PTF in form of a library, which can be used for powermanagement methods. This library is integrated on reader-side. It provides an application interface, which can be used to build a control loop to regulate the provided power of the reader according to the calculation result of the PTF. Furthermore, additional functions are provided by the interface to increase the optimization-possibilities (e.g., getting the current value of the physical relation factor to prevent long and power consuming transmission ranges). This design also makes it possible to integrate this as a hardware component to decrease the calculation time and to be more power efficient.

\section{Case study}

This section describes how the contributed method is implemented and tested. The overview is split into four parts. The first part describes the case study. In the second part, the simulation of the case study and the results are shown. In the third part, the implementation is deployed on real hardware and the measurement results are depicted. The fourth part consists of a measurement of a state-of-the-art mobile NFC-device (smart phone) and the comparison to the results of part two and three to evaluate the benefit to a mobile system.

\subsection{Description of the case study}

In this case study, the PTF-Determinator is implemented in an RFID-System. Furthermore, a feature to restrict the maximum transmission distance $x_{\max }$ between reader and transponder is implemented. When the limit is reached, the system automatically cuts off the power transfer to the transponder to save energy. As a use case the process of reading digital business cards is used. The flow diagram of the use case is shown in Figure 10.

\subsection{Simulation results}

In the first phase, the PTF-Determinator is designed, implemented, and tested using a simulation model for an NFC-System consisting of reader and transponder. The program is an Android application running on an emulator and includes the functionality described in Section 5.1. The SystemC simulation model is based on the target NFC-System but the possible values for $R_{\text {rel }}$ have been modified to show the potential of the method to approximate the physical relation factor over the whole transmission range. The original target hardware has limited possibilities to alter the value $R_{\text {rel }}$. The used simulation model features the possibility to provide the current

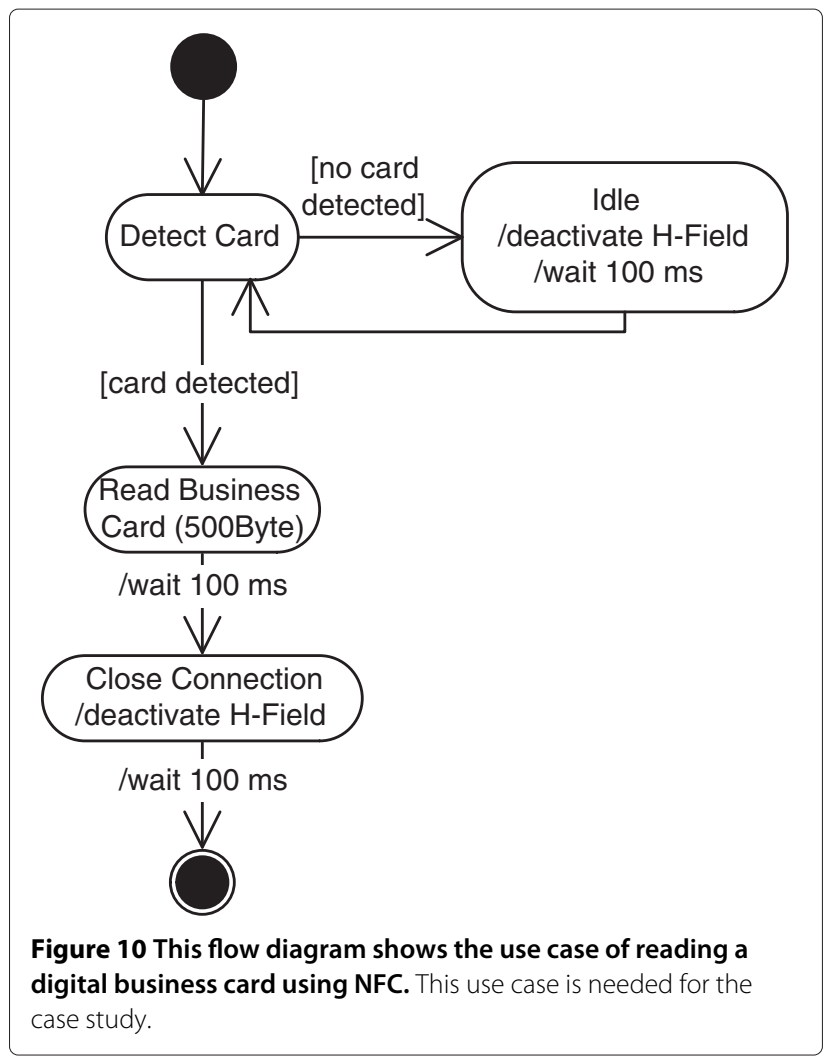

power consumption of its components and the whole system. The model is implemented on transaction layer and is therefore not cycle accurate. The power values are based on the measurement results of the target NFC-Reader and transponder.

The simulation procedure is configured to step through different distances between reader and transponder. The simulator assumes that the two coils are oriented coaxial. The procedure is designed to wait until the execution of the PTF-Determinator with the described use case is finished before the next simulation with another distance is invoked. To deliver realistic results, the use case for reading digital business cards is used.

Figure 11 shows the comparison of the physical and the approximated relation factor of the method between the reader and the transponder. The steps of the approximation depend on the resolution of the $R_{\text {rel }}$ (see Section 4.1). With the modified hardware (more possible values for $R_{\text {rel }}$ ), the physical relation factor can be approximated over the whole transmission range.

In Figure 12, the power consumption is shown when detecting a transponder with and without the PTFDeterminator. The case of $x>x_{\max }$ is shown in Figure 13. The power increase of the central processing unit is simulated using a simple power state machine on reader side. The result shows that the effectiveness of the PTF-evaluation depends on the time relation between 


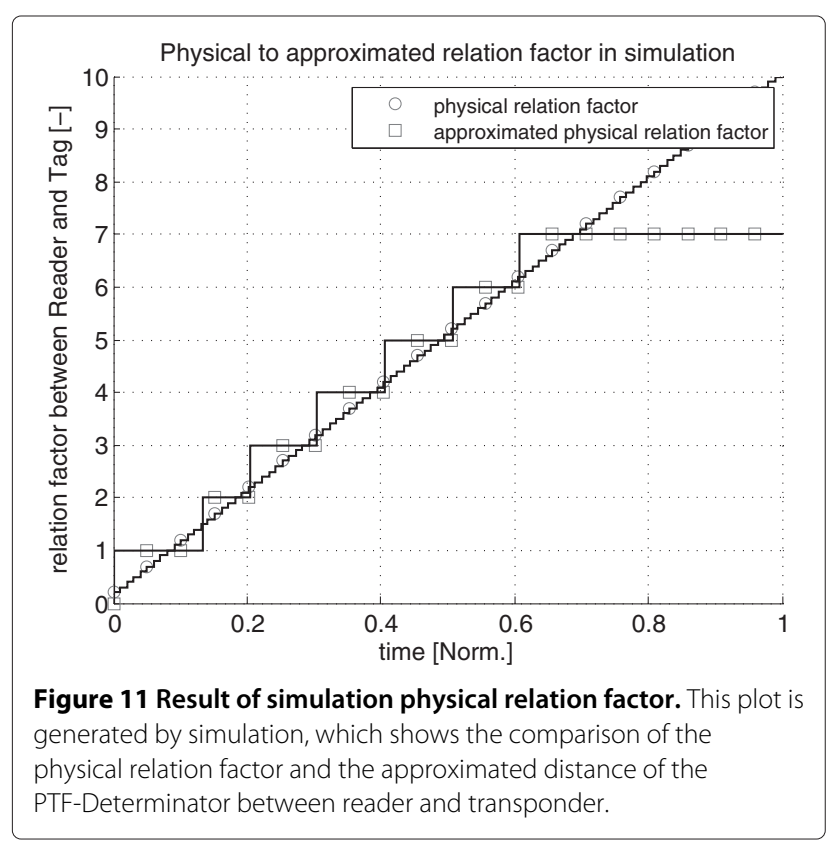

the execution of PTF-Determinator and the use case. Figure 13 shows the result of the simulation in case of the physical relation factor $x>x_{\max }$. If this case occurs, the PTF-Determinator invokes a forced cut-off in the power transfer on reader side to the transponder. With this method an energy wastage is prohibited. A comparison of the saved energy in relation to the simulated distance $d$ between reader and transponder is shown in Table 2.

The saved transmission energy (energy needed by the Reader-IC for the power transfer to the transponder) is as high as $80 \%$ in close distance. Thus, this approach offers a lot of potential in saving energy. This result depends on the use case and can only be achieved by adapting the hardware and no further influence is given by the environment. The benefit of saving energy decreases if the physical relation increases because the transmission power has to be increased to provide enough power for the transponder. If the physical relation factor $x$ is higher than $x_{\max }$, then the power supply is cut off as described.
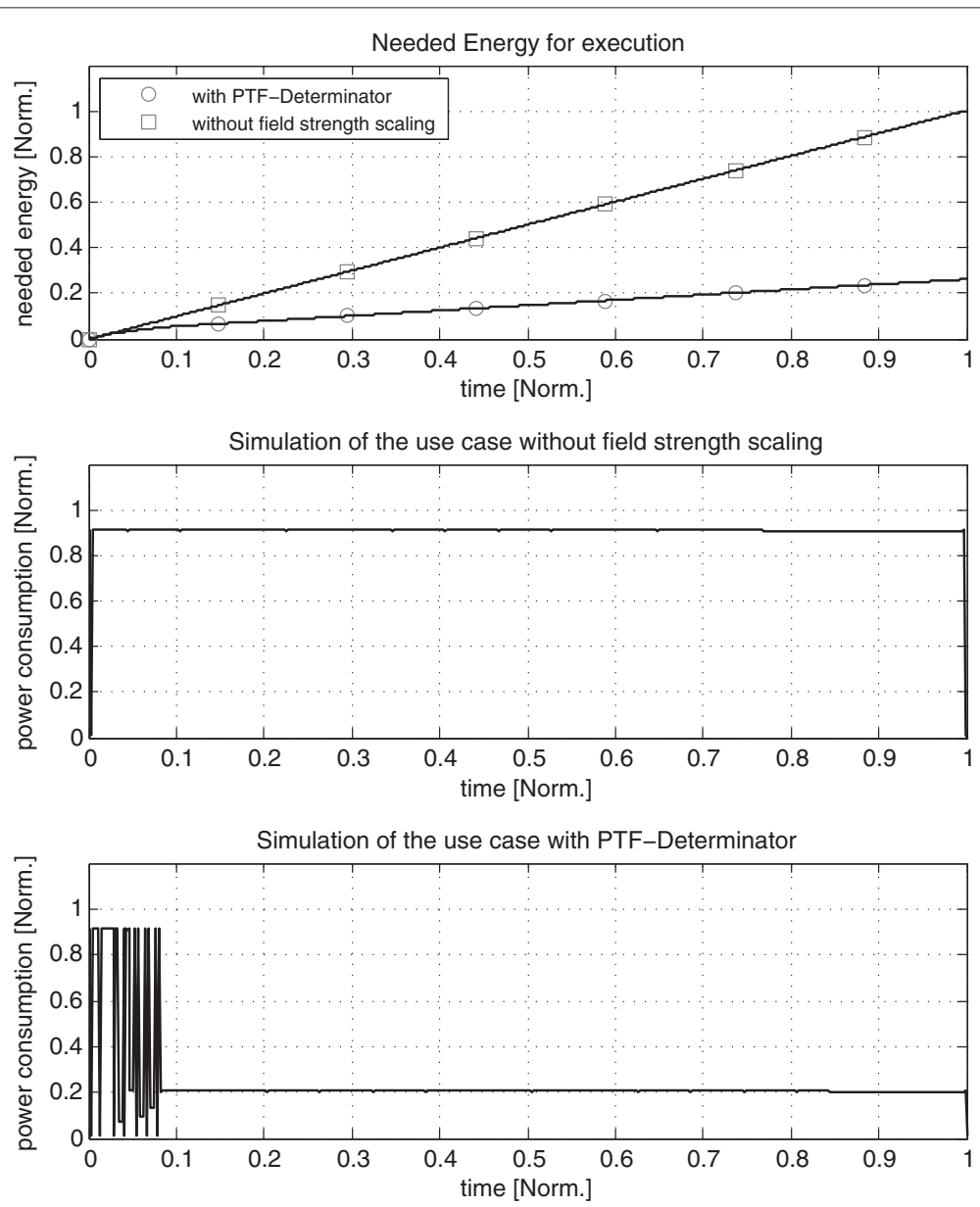

Figure 12 Result of $\operatorname{simulation} \boldsymbol{x}<\boldsymbol{x}_{\max }$. The plot shows the simulation result, with the use case described in Figure 10, with and without an improved version of the PTF-Determinator from [1] under the condition $x<x_{\max }$. 

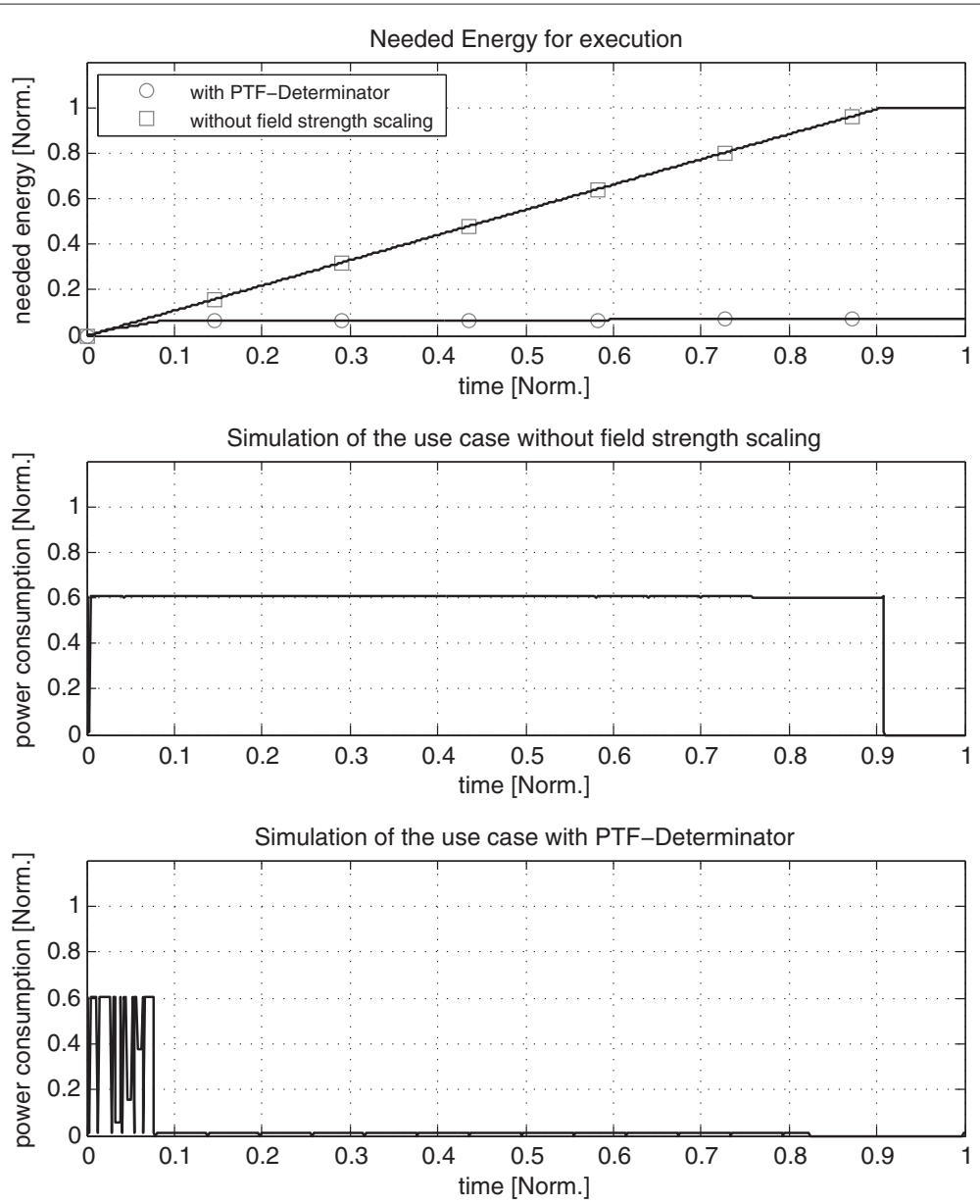

Figure 13 Result of simulation $\boldsymbol{x}>\boldsymbol{x}_{\mathbf{m a x}}$. The plot shows the simulation result, with the use case described in Figure 10, with and without an improved version of the PTF-Determinator from [1] under the condition $x>x_{\max }$.

This leads to a power saving of $93 \%$ with the disadvantage of loosing connectivity to the transponder. To reestablish a communication, the transponder's current physical relation factor has to be below the maximum allowed physical relation factor.

\subsection{Measurement results}

In the second phase, the PTF-Determinator is implemented and tested on real hardware. Power consumption measurements are conducted for verification purposes. The program is the same used during the simulation and includes the functionality described in Section 5.1. The used set-up is shown in Figure 14 and described in Table 3 . To verify the method and compare the resulting power consumption of the simulation and a real environment behavior, the method is deployed and tested on a target NFC-System. To get the needed measurement data, the hardware is placed into a hardware-inthe-loop measurement suite. The suite is configured to acquire the power consumption of the whole system while the program under test is executed. For comparison, the use case of reading a digital business card without the PTF-Determinator is also measured. The physical relation between reader and transponder is altered to validate the functionality of the program and to evaluate its influence on its power consumption.

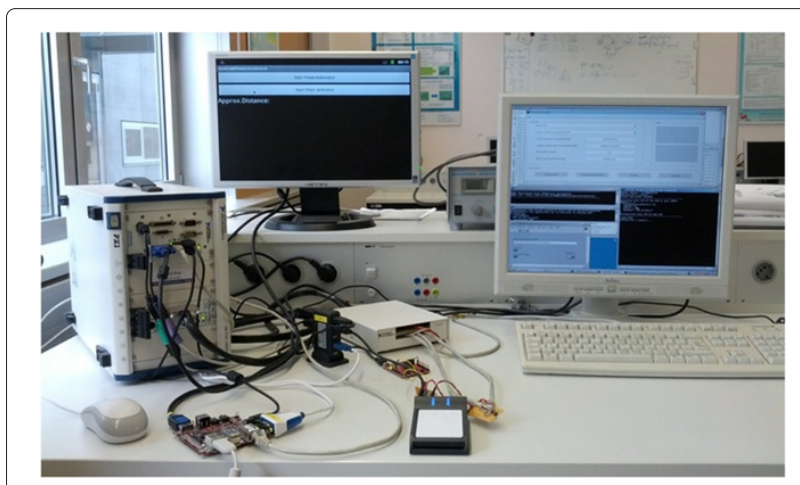

Figure 14 Setup used for the measurement of the use case. It consists of the development board, the RFID-Reader, the measurement device from National Instruments and an evaluation software in Matlab. 
Table 3 The setup used for the power measurement needed for the case study

\begin{tabular}{ll}
\hline Program language & Java \\
Development board & Beagleboard \\
Operating system & Android 2.3.4 \\
NFC-Reader & USB-Reader connected \\
& to the development board \\
Measurement device & Hardware-in-the-loop \\
& Measurement-suite \\
\hline
\end{tabular}

Figure 15 shows the power consumption of the NFCSystem with and without the PTF-Determinator. In Figure 16, the power consumption of the NFC-System is shown when physical relation factor $x>x_{\max }$. This leads to cutting off the power supply of the transponder. This cut-off prevents the energy waste invoked by the power loss in the reader circuit and the loosely coupled power transfer to the transponder. The measurement results also show that the influence of the physical relation factor on the consumed energy, invoked by the influence of the reader and transponder coil, has to be considered. The execution of the PTF-Determinator results in a small overhead as shown in Figures 15 and 16.

In Table 4, a comparison between the simple card detection and the usage of the PTF-Determinator is made. It includes the needed energy of the whole procedure. The needed energy is compared and the saved energy can be evaluated when using the PTF-Determinator instead of the simple card detection. The approximation of the physical relationship is limited on the real hardware, because only few steps to scale the magnetic field are supported. However, it can be shown that the needed energy for the power transmission is $26 \%$ lower when considering the execution of the PTFDeterminator in case of $x<=x_{\max }$. The needed energy in case of $x>x_{\max }$ for the power transmission is about $75 \%$ lower compared to the use case without PTF-Determinator.
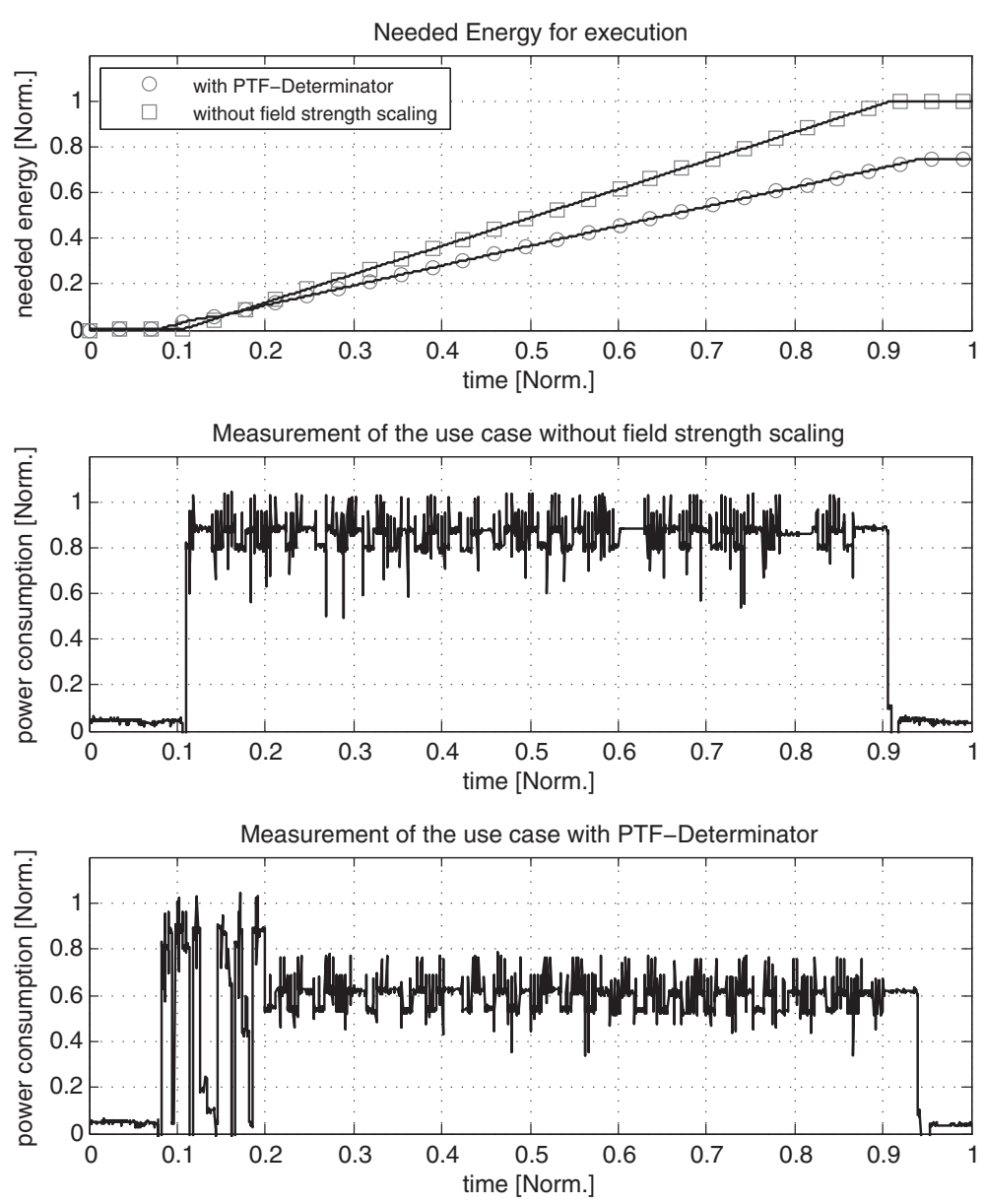

Figure 15 Result of the measurement $x<x_{\max }$. The plot shows the measurement result, with the use case described in Figure 10, with and without an improved version of the PTF-Determinator from [1] under the condition $x<x_{\max }$. 

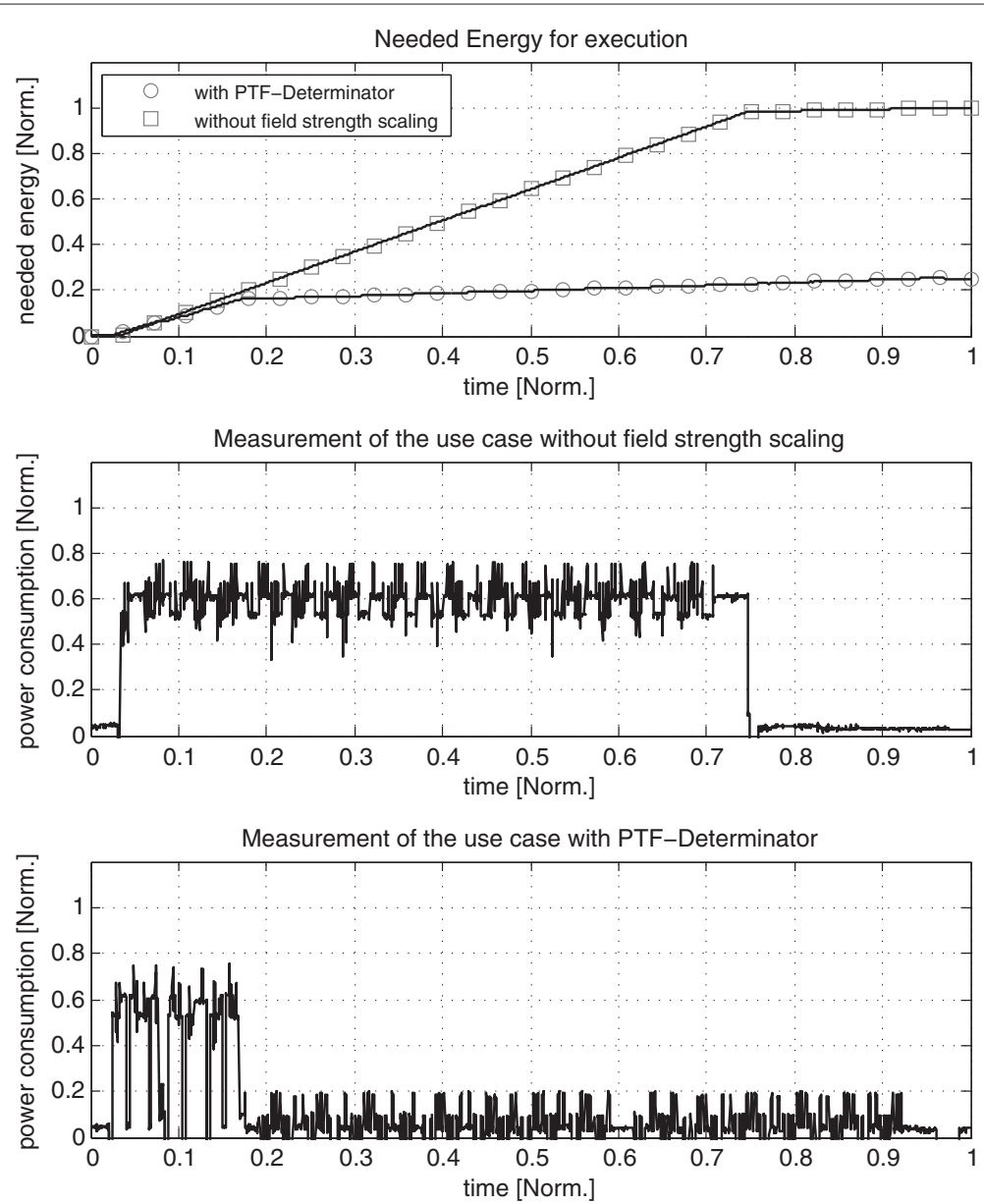

Figure 16 Result of the measurement $\boldsymbol{x}>\boldsymbol{x}_{\mathbf{m a x}}$. The plot shows the measurement result, with the use case described in Figure 10, with and without an improved version of the PTF-Determinator from [1] under the condition $x>x_{\max }$.

\subsection{Comparison to an NFC-enhanced smart phone}

In this section, a power measurement of a state-of-theart smart phone is done. The idea is to get results, how field strength scaling is influencing the power consumption of such a device. The power consumption is measured directly at the battery source and therefore includes all components (e.g., display and processor). The measurement setup is listed in Table 5. The results of the simulation in Section 5.2 and the measurement in Section 5.3 can then be combined with this measurement to one result to get an approximation of the possible power reduction

Table 4 Comparison of the saved energy for the RFID-Transmission in percent between the method with and without an improved version of the PTF-Determinator from [1] in the measurement

\begin{tabular}{llll}
\hline Physical & $\begin{array}{l}\text { Energy with PTF } \\
\text { (Norm.) }\end{array}$ & $\begin{array}{l}\text { Energy without PTF } \\
\text { (Norm.) }\end{array}$ & $\begin{array}{l}\text { Energy saved } \\
\text { (\%) }\end{array}$ \\
\hline$x<=x_{\max }$ & 0.745 & 1.000 & 25.51 \\
$x>x_{\max }$ & 0.251 & 1.000 & 74.88 \\
\hline
\end{tabular}

using field strength scaling on a state-of-the-art smart phone.

To achieve the evaluation goal, the power consumptions of the different smart phone states concerning the ReaderIC (RFID) (e.g., "reading the tag" or "RFID is powering the transponder") are extracted from the power measurement. The power consumption of the smart phone during the state idle and reading the transponder (tag) is shown in Figure 17. It can be seen that there is a significant

Table 5 The measurement setup for the acquisition of the smart phone's power consumption including the devices used for the measurement

\begin{tabular}{ll}
\hline Smart phone & Samsung Nexus S \\
Operating system & Android 2.3.4 \\
Application & NFC Taglnfo from \\
& NFC Research Hagenberg \\
Measurement device & PXI NI 6221 using DAQ SignalExpress \\
Transponder & Infineon Tag Type 2 (2kB) \\
\hline
\end{tabular}



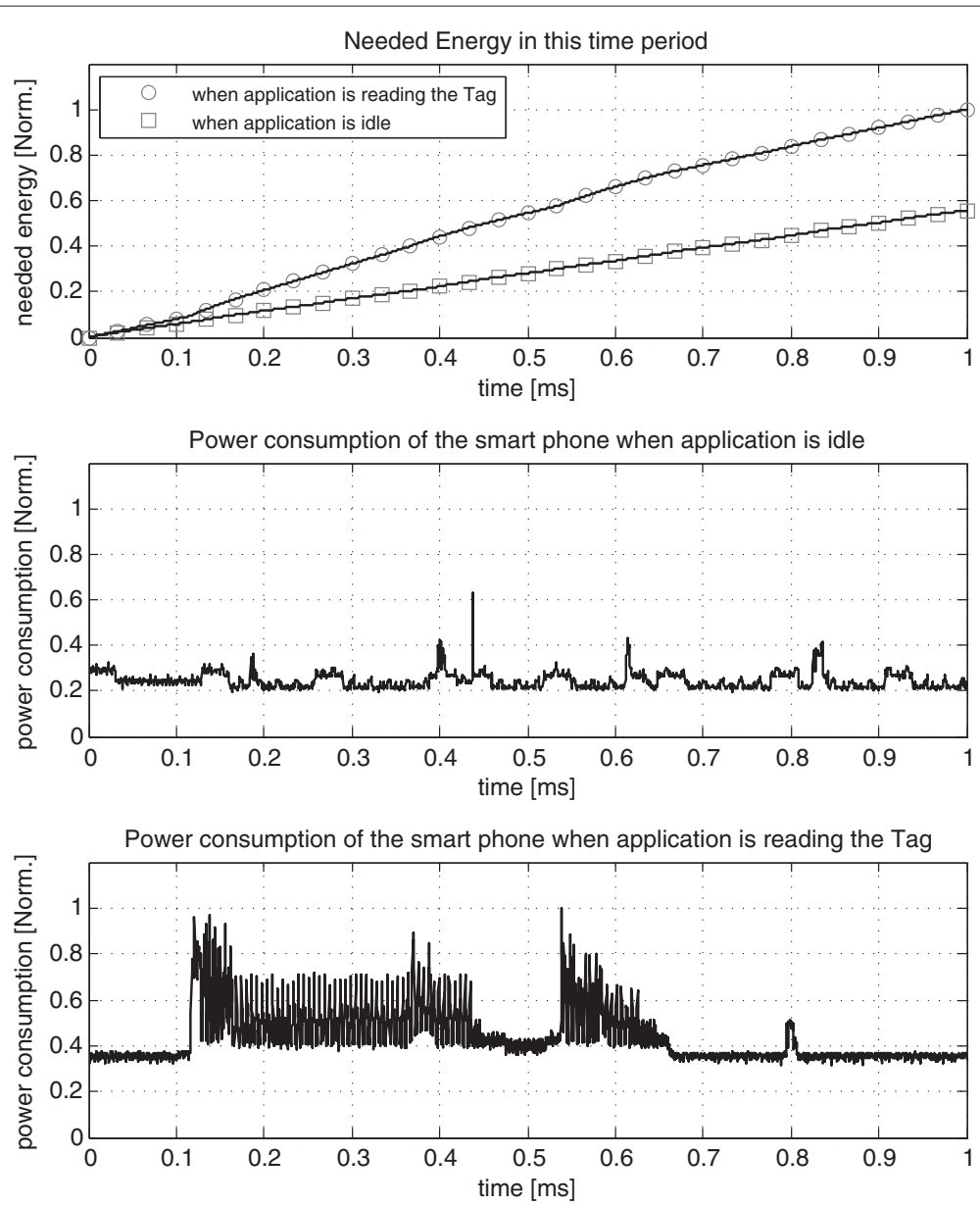

Figure 17 Power consumption of the state-of-the-art smart phone with NFC. This figure shows the power consumption during the execution of the application described in the measurement setup (see Table 3). The figure is divided into three plots, showing the energy consumption as well as the power consumptions in idle state and during the smart phone's reading process of the transponder.

smart phone power consumption rise during the process of reading the transponder. Table 6 shows this different power consumptions of the smart phone.

The extraction shows that during the reading process of the transponder the power consumption increases up to $107 \%$ compared to the idle state power consumption. Furthermore, the state while the transponder is powered by the Reader-IC without executing any operation consumes $50 \%$ more power. The presented numbers show

Table 6 Results of the state-of-the-art smart phone's measurement are presented in form of its power consumptions during different states

\begin{tabular}{ll}
\hline State & Consumed power [\%] \\
\hline NFC is on but no transponder in range & 100 \\
Transponder is powered over NFC & 150 \\
Transponder is powered over NFC & 207 \\
and reading operations are performed & \\
\hline
\end{tabular}

that reducing this power consumption has potential of saving energy in the whole system (smart phone).

These results of a state-of-the-art smart phone are now used to scale the results ( $\left.E_{\text {unscaled }}\right)$ of Sections 5.2 and 5.3 to get an approximation of the expectable results $\left(E_{\text {scaled }}\right)$ of field strength scaling on a state-of-the-art smart phone using Equation 6.

$$
E_{\text {scaled }}=100-\frac{\left(100-E_{\text {unscaled }}\right) \cdot 1,07+100}{207} \cdot 100
$$

Theses combined results (transmission power saving and the power states of the art smart phone) show that approximately $41 \%$ of the energy can be saved compared to simulation with a modified hardware and $13 \%$ can be expected in a real environment without changing the hardware. In case of $x>x_{\max }$ the approximated saved energy is as high as $48 \%$ in simulation and $39 \%$ in a real environment. 


\section{Conclusion}

The additional energy consumption by using RFID in mobile system is a challenging task. This article shows that magnetic field strength scaling in HF-Band RFIDSystems is a good way to reduce this energy wastage. The three investigations made are a way to deal with the challenges of the dynamic behavior during communication like the changing physical relation factor (e.g., the user is moving the transponder towards the reader). This study has also shown that the integration of the run-time method to determine the PTF on reader side is feasible. The energy saving potential of the presented PTF-Determinator, using the PTF to scale down the magnetic field strength, is shown by simulation and is verified through measurement. In simulation using a improved hardware model (more suitable steps to scale the magnetic field strength are included) up to $80 \%$ less transmission energy is needed. In the measurement, using existing hardware, the energy needed by the transmission is reduced by $26 \%$ compared to a simple carddetection method. Thus, a state-of-the-art NFC smart phone featuring our proposed method would reduce the battery drain by up to $13 \%$. The implemented feature to set a maximum physical relation factor, by cutting off the power supply to avoid energy-consuming transactions, has been proven by measurement to reduce the transmission energy by up to $75 \%$. This results into a reduction of $39 \%$ less battery drain of a state-of-the-art smart phone.

The combination of simulation and verification through measurement in one tool chain has proven to be a good way for developing power-aware systems. The simulation gives the opportunity of reconfiguring the hardware (design hardware and software together) to evaluate the potential of ideas like magnetic field strength scaling.

In future work, the proposed PTF-Determinator method shall be improved, focusing on optimizing the approximation of the physical relation factor. Furthermore, the other investigations made regarding the dynamic field strength scaling during communication and the usage in an environment with multiple transponders will be verified by simulation and measurement.

\section{Competing interests}

The authors declare that they have no competing interests.

\section{Acknowledgements}

We would like to thank our industrial partners Infineon Technologies Austria AG as well as Enso Detego GmbH for their support. Furthermore, we would like to thank the Austrian Federal Ministry for Transport, Innovation, and Technology, which funded the project META[:SEC:] under the FIT-IT contract FFG 829586.

\footnotetext{
Author details

${ }^{1}$ Graz University of Technology, Graz, Austria. ${ }^{2}$ Infineon Technologies Austria AG, Graz, Austria
}

Received: 30 October 2012 Accepted: 18 March 2013

Published: 16 April 2013

\section{References}

1. M Menghin, N Druml, C Steger, R Weiss, J Haid, H Bock, in Fourth International EURASIP Workshop on RFID Technology (EURASIP RFID) 2012. The PTF-Determinator: a run-time method used to save energy in NFC-Systems (Turin, 2012), pp. 92-98

2. M Menghin, N Druml, C Steger, R Weiss, J Haid, H Bock, in 5th International Workshop on Near Field Communication (NFC 2013). NFC-DynFS: a way to realize dynamic field strength scaling during communication (Zurich, 2013), pp. 1-6

3. D Cheng, Z Wang, Q Zhou, in 4th International Conference on Wireless Communications, Networking and Mobile Computing (WiCOM'08). Analysis of distance of RFID systems working under 13.56 MHz (Dalian, 2008), pp. 1-3

4. J Clulow, GP Hancke, MG Kuhn, T Moore, in Third European conference on Security and Privacy in Ad-Hoc and Sensor Networks (ESAS'06). So, near and yet so far: distance-bounding attacks in wireless networks (Springer, New York, 2006), pp. 83-97

5. $X X u, L$ Gu, J Wang, $G$ Xing, in IEEE International Conference on Pervasive Computing and Communications (PerCom 2010). Negotiate power and performance in the reality of RFID systems (Mannheim, 2010), pp. 88-97

6. Wireless Power Consortium. System Description Wireless Power Transfer, Vol. I, Part 1, (2011), p. 32. http://www.wirelesspowerconsortium.com/ downloads/wireless-power-specification-part-1.html. Accessed 11 April 2013

7. M Wendt, M Grumer, C Steger, R Weiss, U Neffe, A Muehlberger, in Proceedings of the 2008 ACM Symposium on Applied Computing (SAC'08). System level power profile analysis and optimization for smart cards and mobile devices (ACM, New York, 2008), pp. 1884-1888. doi:10.1145/1363686.1364144

8. A Genser, C Bachmann, C Steger, R Weiss, J Haid, in IEEE Asia Pacific Conference on Circuits and Systems (APCCAS 2010). Estimation-based run-time power profile flattening for RF-powered smart card systems, (Kuala Lumpur, 2010), pp. 1187-1190

9. T Lohmann, M Schneider, C Ruland, in Smart Card Research and Advanced Applications, vol. 3928 of Lecture Notes in Computer Science, ed. by J Domingo-Ferrer, J Posegga, and D Schreckling. Analysis of power constraints for cryptographic algorithms in mid-cost RFID tags (Springer, Berlin, 2006), pp. 278-288. doi:10.1007/1173344720

10. J Mercier, C Dufaza, M Lisart, in Proceedings of the 2007 International Symposium on Low Power Electronics and Design (ISLPED'07). Signoff power methodology for contactless smartcards (ACM, New York, 2007), pp. 407-410. doi:10.1145/100000

11. E Rolf, $V$ Nilsson. Near field communication (NFC) for mobile phones (Master's Thesis, Lund University, 2006), p. 25. http://www.es.Ith.se/teorel/ Publications/TEAT-5000-series/TEAT-5082.pdf. Accessed 11 April 2013

12. N Druml, M Menghin, C Steger, R Weiss, A Genser, J Haid, in 15th Euromicro Conference on Digital System Design (DSD 2012). Adaptive field strength scaling - a power optimization technique for contactless reader/ smart card systems (Izmir, 2012), pp. 616-623

13. N Kamineni, X Li, in International Conference on Computing Communication and Networking Technologies (ICCCNT 2010). Analysis of anti-collision multi-tag identification algorithms in passive RFID systems (Karur, 2010), pp. 1-8

14. DF Tseng, ZC Lin, Anti-collision algorithm with the aid of interference cancellation and tag set partitioning in radio-frequency identification systems. IET Commun. 3, 143-150 (2009)

15. KWu, Y Liu, in Second International Conference on Future Networks (ICFN'10). A new energy-aware scheme for RFID system based on ALOHA (Sanya, Hainan, 2010), pp. 149-152

16. J Liu, W Tong, in IEEE International Conference on Cloud Computing and Intelligence Systems (CCIS 2011). Dynamic share energy provisioning service for one-hop multiple RFID tags identification system, (Beijing, 2011), pp. 342-347

17. J Haid, W Kargl, T Leutgeb, D Scheiblhofer, in Proceedings of Telecommunications and Mobile Computing Graz Series (TCMC 2005). Power management for RF-powered vs. battery-powered devices, (Graz, 2005) 
18. A Roy, SM Rumble, R Stutsman, P Levis, D Mazières, N Zeldovich, in Proceedings of the sixth conference on Computer systems, (EuroSys'11). Energy management in mobile devices with the cinder operating system (ACM, New York, 2011), pp. 139-152. doi:10.1145/1966445.1966459

19. K Finkenzeller, RFID Handbook: Fundamentals and Applications in Contactless Smart Cards and Identification, 2nd edn. (Wiley, New York, 2003)

20. XXu, L Gu, J Wang, G Xing, SC Cheung, Read more with less: an adaptive approach to energy-efficient RFID systems. IEEE J. Sel. Areas Commun. 29(8), 1684-1697 (2011)

21. W Rankl, W Effing, Smart Card Handbook: Physical and Electrical Properties, 3rd edn. (Wiley, New York, 2003)

doi:10.1186/1687-3963-2013-4

Cite this article as: Menghin et al:: Using field strength scaling to save energy in mobile HF-band RFID-systems. EURASIP Journal on Embedded Systems 2013 2013:4.

\section{Submit your manuscript to a SpringerOpen ${ }^{\circ}$ journal and benefit from:}

- Convenient online submission

- Rigorous peer review

- Immediate publication on acceptance

- Open access: articles freely available online

- High visibility within the field

- Retaining the copyright to your article

Submit your next manuscript at $\boldsymbol{~ s p r i n g e r o p e n . c o m ~}$ 